\title{
A post-mortem population survey on foetal-infantile end-of-life decisions: a research protocol
}

\author{
Laure Dombrecht ${ }^{1 *}$ (D) Kim Beernaert ${ }^{1}$, Ellen Roets ${ }^{2}$, Kenneth Chambaere ${ }^{1}$, Filip Cools ${ }^{3}$, Linde Goossens ${ }^{4}$, \\ Gunnar Naulaers ${ }^{5}$, LuC De Catte ${ }^{6}$, Joachim Cohen ${ }^{1+}$, LuC Deliens ${ }^{1+}$ on behalf of the NICU consortium ${ }^{7,8,9,10,11}$
}

\begin{abstract}
Background: The death of a child before or shortly after birth is frequently preceded by an end-of-life decision (ELD). Population-based studies of incidence and characteristics of ELDs in neonates and infants are rare, and those in the foetal-infantile period ( $>22$ weeks of gestation -1 year) including both neonates and stillborns, are nonexistent. However, important information is missed when decisions made before birth are overlooked. Our study protocol addresses this knowledge gap.

Methods: First, a new and encompassing framework was constructed to conceptualise ELDs in the foetal-infantile period. Next, a population mortality follow-back survey in Flanders (Belgium) was set up with physicians who certified all death certificates of stillbirths from 22 weeks of gestation onwards, and infants under the age of a year. Two largely similar questionnaires (stillbirths and neonates) were developed, pilot tested and validated, both including questions on ELDs and their preceding decision-making processes. Each death requires a postal questionnaire to be sent to the certifying physician. Anonymity of the child, parents and physician is ensured by a rigorous mailing procedure involving a lawyer as intermediary between death certificate authorities, physicians and researchers. Approval by medical societies, ethics and privacy commissions has been obtained.
\end{abstract}

Discussion: This research protocol is the first to study ELDs over the entire foetal-infantile period on a population level. Based on representative samples of deaths and stillbirths and applying a trustworthy anonymity procedure, the research protocol can be used in other countries, irrespective of legal frameworks around perinatal end-of-life decision-making.

Keywords: End-of-life decisions, Neonates, Stillbirths, Termination of pregnancy, Mortality follow-back survey, Population-based

\section{Background}

Recent decades have seen an increase in possible medical and technical interventions for critically ill neonates and infants [1]. However, in Flanders, Belgium about 8.7 per thousand children still die during the foetal-infantile period, i.e. from foetuses of more than $500 \mathrm{~g}$ or 22 weeks of gestation up until 1 year after birth [2]. This is comparable with death rates reported, for instance, in the United States [3]. Many of these deaths occur at

\footnotetext{
* Correspondence: Laure.Dombrecht@ugent.be

${ }^{\dagger}$ Joachim Cohen and Luc Deliens contributed equally to this work.

${ }^{1}$ End-of-Life Care Research Group, Ghent University \& Vrije Universiteit Brussel (VUB), Ghent, Belgium

Full list of author information is available at the end of the article
}

neonatal intensive care units (NICUs) and are preceded by a possibly life-shortening end-of-life decision (ELD) [4-6]. In neonates, these include non-treatment decisions such as withholding or withdrawing life-sustaining treatment, intensification of alleviation of pain and/or other symptoms with a potential life-shortening effect and intentionally ending life with lethal drugs [7]. Additionally, prenatal diagnostic techniques (genetic techniques, prenatal imaging techniques) have evolved considerably, leading to an increasing number of congenital malformations being diagnosed prenatally instead of after birth [8,9]. Some decisions such as abstinence from treatment [8-10] or termination of pregnancy

(c) The Author(s). 2018 Open Access This article is distributed under the terms of the Creative Commons Attribution 4.0 International License (http://creativecommons.org/licenses/by/4.0/), which permits unrestricted use, distribution, and reproduction in any medium, provided you give appropriate credit to the original author(s) and the source, provide a link to the Creative Commons license, and indicate if changes were made. The Creative Commons Public Domain Dedication waiver (http://creativecommons.org/publicdomain/zero/1.0/) applies to the data made available in this article, unless otherwise stated. 
(TOP) $[8,9]$ can be made during gestation in cases of the detection of serious abnormalities [11-13]. For stillbirths from 22 weeks of gestation and onwards which is considered as the definition of a viable foetus by the WHO - TOPs are considered late terminations. Stillborns and deceased neonates cannot be seen as separate patient populations, since they are in essence the same patient where an ELD can be made either before or after birth. The only difference is therefore the occurrence of birth and not necessarily a difference in disorders or congenital anomalies. Research into end-of-life decision-making on a population level should therefore take into account the foetal-infantile period in its entirety (instead of both periods separately). This is needed to provide reliable incidence rates and information on the decision-making process in this vulnerable population. Evaluation and monitoring of ELD practice in the entire foetal-infantile period could lead to better understanding of current prenatal and neonatal health care and detect points of improvement since there have been no all-inclusive guidelines up to the present.

Population-based studies (i.e. with all death cases as the focus) are ideal to study the incidence and characteristics of ELDs, but such studies are rare in neonates and infants [14-16] and, to our knowledge, non-existent in stillborns. In neonates, results are mostly based on reviews of medical records of a NICU at a particular hospital. In these studies 40 to $93 \%$ of deaths in a NICU follow withdrawal of life-sustaining treatments [6, 1719]. The larger scale EURONIC study was based on physicians' self-reported practices within 143 European NICUs in the 1990s [20]. The only population-based studies are from the Netherlands (in 2014) [15] and Belgium (in 2000) [14]. These studies found an ELD being made in $60 \%$ of all deaths of neonates and infants. In stillborns, previous studies in 2003 [11] and in 20002005 [13] have only looked at the prevalence of late TOP $[11,13,21]$. Not much is known about the entirety of end-of-life practices (including decisions other than TOP) and their decision-making process, or about patient characteristics besides gestational age and the presence of foetal anomalies.

We developed a study design to evaluate and monitor ELDs and their decision-making process across the entire foetal-infantile period in Flanders, Belgium. The study design involves the development of a validated conceptual framework of ELDs spanning the entire foetal-infantile period (based on existing frameworks) and the development of a survey methodology that addresses the particular difficulties in capturing and surveying stillbirths and neonatal deaths, and provides opportunities for comparison of ELD practices between hospitals.

\section{Methods}

This population study has the design of a mortality follow-back survey based on all death certificates of stillbirths and neonates. Questionnaires are either sent to the certifying physicians by post or are provided at maternity wards. In order to develop these questionnaires, adjustments to an existing neonatal ELD framework needed to be made.

\section{Conceptual framework of foetal-infantile ELDs}

Prenatal ELDs should be taken into account when presenting a reliable and complete picture on foetal-infantile ELD practices. However, to date these prenatal ELDs have not been included in a comprehensive framework with neonatal ELDs. We adjusted a previously existing and validated framework of ELDs in neonates [7] in order to include both prenatal and neonatal ELDs. This framework [7] includes three dimensions: 'medico-technical', 'medico-ethical' and 'consultation with parents'. The dimension 'consultation with parents' was excluded from our own framework since no decision can be made prenatally without at least the mother consenting to an intervention. Furthermore, the dimension 'consultation with patients' is also excluded from the adult ELD framework where the medical decision and its intention are the only determinants of an ELD. However, this dimension is still very important which is why consultation with parents will still be addressed in detail by means of additional questions outside the ELD framework. These encompass the following:

1. The medico-technical classification or medical acts $[7,22]$ :

- non-treatment decisions such as withholding or withdrawal of life-sustaining treatment

- administering drugs or medical interventions

2. The medico-ethical classification or the lifeshortening intention of the physician can be [7, 14]:

- no intention but taking into account a potentially life-shortening effect

- the potentially life-shortening effect is not the main goal but partly intended (co-intention)

- an explicit life-shortening intention.

To cover all possible decisions that could possibly influence the death of a foetus or infant, both dimensions should be taken into consideration. As a side note, intentionally ending the life of a child is illegal, meaning that in this case, the medico-ethical dimension is 
considered to be all the more important since no emphasis is put on the medico-technical classification specifically.

We presented this framework for validation to gynaecologists in eight individual interviews and two expert panels representing seven different hospitals. The gynaecologists were asked to give clinical examples for all possible ELD categories applied to the prenatal context, and to add more categories in case any were missing. As soon as a realistic example was given and agreed on by others, that ELD was considered possible and included in the framework (Table 1). The resulting foetal-infantile ELD framework was then thoroughly reviewed by three neonatologists.

Literature on end-of-life practices prior to stillbirth distinguishes between non-aggressive obstetric management and TOP [9, 10, 23]. Non-aggressive obstetric management (or abstinence from treatment) is the denial of interventions which are needed to sustain the life of the foetus because of a poor foetal prognosis [8-10]. TOP however, actively ends foetal life $[8,9]$ by preterm induction of labour either with or without feticide (administering medication to intentionally end the life of the foetus before birth) prior to the termination [24].

\section{Questionnaires}

Based on this adjusted framework, two separate but similar questionnaires were developed for ELDs in stillborns and ELDs in neonates respectively, since both populations have their own specificities. Both questionnaires include questions about ELDs, the decision-making process, the involvement of parents in this process, the involvement of colleagues and experts, and the ELD policy of the hospital.

For neonates and infants, previously validated questionnaires that focus on end-of-life decisions in minors and neonates $[14,16,25]$ were used as the basis for our questionnaire. We mainly focused on updating the terms and grammar used, term ambiguity, length of the questionnaire and comparability to the previous ELD study [14]. The resulting questionnaire was thoroughly pilot tested and validated with eight neonatologists who

Table 1 A comprehensive framework of end-of-life practices in the foetal-infantile period

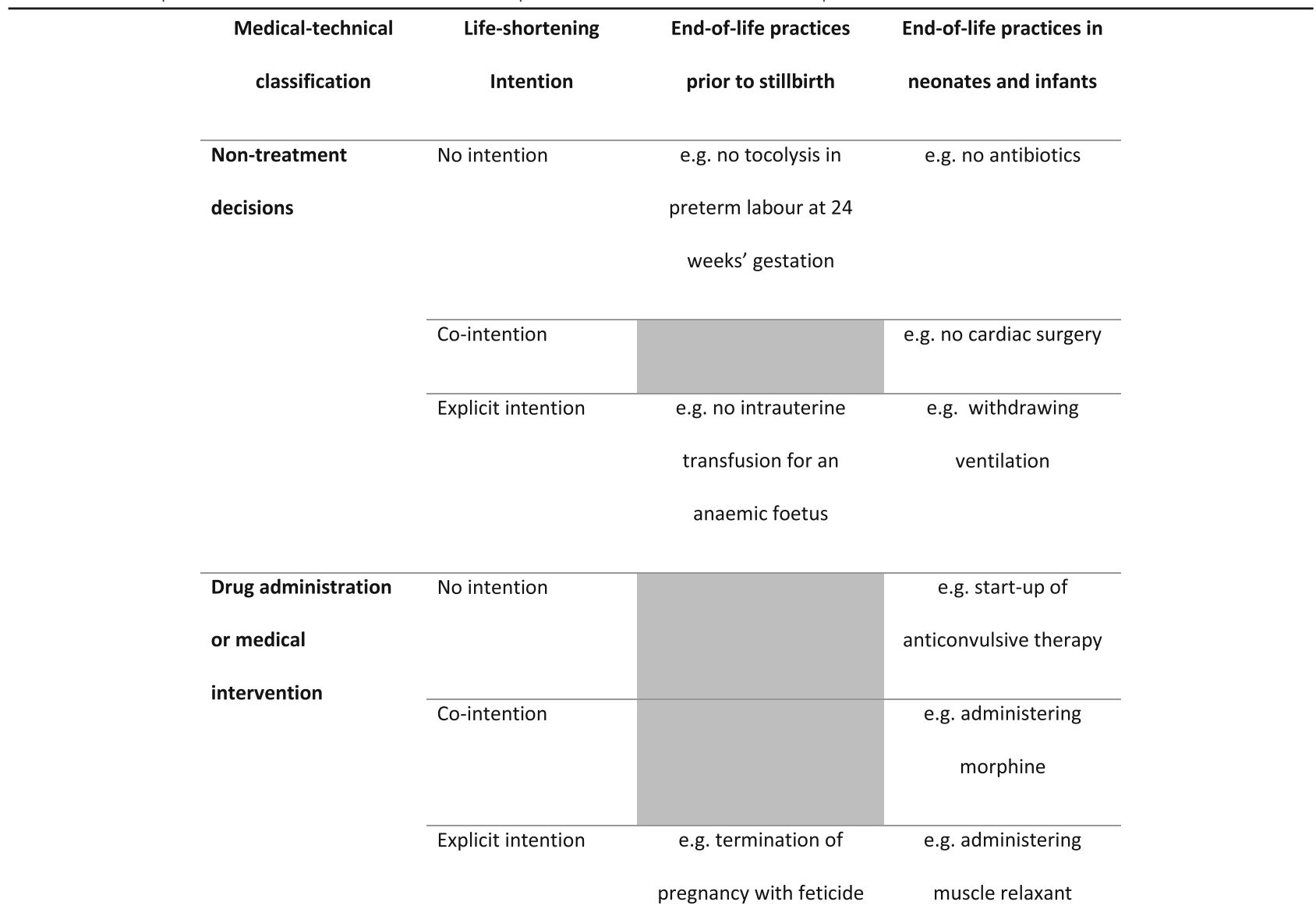

Indicated in grey: not valid in the prenatal context. NTD: non-treatment decisions, APS: alleviation of pain and/or symptoms, Li: lethal interventions. This table only includes medical interventions to the child or foetus 
represented all eight Flemish NICUs, researchers in the field of end-of-life care and an ethicist.

For ELDs in stillborns a new questionnaire was developed based on previously validated questionnaires on TOP after 22 weeks $[11,12]$, questionnaires on ELDs in minors and neonates $[14,16,26]$, and the newly developed framework for end-of-life practices in the foetal-infantile period. This questionnaire was thoroughly pilot tested and validated with eight gynaecologists, three neonatologists, researchers in the field of end-of-life care, an ethicist and a lawyer in the field of end-of-life care.

Neither questionnaire asks directly about categories of ELDs but classifies these based on a series of core questions following the two dimensions of the conceptual framework about 1) which act or omission was used (medico-technical), and 2) which life-shortening intention was associated with the act (medico-ethical). Additional questions were asked about the ways in which parents were involved in the decision-making process (parent consultation).

\section{Population and setting}

The population includes: all stillbirths from 22 weeks of gestation or more and/or a birthweight of $500 \mathrm{~g}$ or higher (i.e. the internationally acknowledged limit of viability of the foetus $[24,27,28]$ ) and all deceased neonates and infants under the age of 1 year occurring in Flanders and Brussels where the mother is a Flemish resident. No sample is drawn; the full population is included over a data collection period of 12 months for stillbirths and 16 months for neonates and infants. The longer observation period for neonate and infant deaths was chosen because these deaths are less common than late termination stillbirths [2] and we wanted to obtain a population large enough to make reliable prevalence estimates of end-of-life practices.

Deaths to be included in the study are identified using the death certificate. Every death of a Flemish resident in Flanders and Brussels must be declared by means of a death certificate to the Flemish Agency for Care and Health of the Ministry of the Flemish Community or the Brussels Health and Social Observatory respectively. The physician, in our study most probably a neonatologist, paediatrician or gynaecologist, completes the main part of the death certificate which indicates the sex of the child, the date of birth and the date of death, medical information such as the cause of death, whether or not the child was alive at the time of birth, and the time and place of death [29]. The physician then signs the certificate and adds his or her medical registration number. The death certificate is then sent to the civil registrar of the municipality where the death took place where additional information is completed on the death certificate such as socio-demographic information about the child and its parents. Certificates are then processed by the provinces before being sent to the central administration authorities. It can take up to 3 months for death certificates to reach these administration authorities.

\section{Design and procedures}

A mortality follow-back procedure is followed, slightly modifying well-established procedures in adults [22] and minors [26]. Modifications concern a more stringent anonymity procedure and an alternative identification procedure for stillbirths between 22 and 26 weeks. As for the anonymity procedure, ethical and legal considerations (criminal prosecution is possible for reported illegal ELDs) make it necessary to pay greater attention to the protection of confidentiality of the physician, to the privacy of the deceased, the parents and the relatives, and to the security of the data that will be obtained in the survey. By ensuring total anonymity, both the response rate and the reliability of the responses can be improved. The different stages of the survey i.e. the mailing, receiving and processing of the questionnaires will be separated and performed by four separate entities (see Fig. 1).

1. The death certificate administration authorities (namely Flemish Agency for Care and Health of the Ministry of Welfare, Health and Family of the Flemish Government) is responsible for construction and management of the mailing database and the mailing of the questionnaires. Each case is ascribed a unique coded number derived from the death certificate number. These unique numbers are used at the end of the study to link the questionnaires to the demographic and morbidity data (such as ICD-10 codes of the cause of death) of the deceased, derived from the death certificates, in a database provided by the administration authorities. An accompanying letter is included with the questionnaire providing the physician with enough patient characteristics to identify the patient. These include sex, date of (still)birth, date of death and municipality of death; for stillborns the date of death is replaced by the date of birth of the mother. When the lawyer (see below) receives the questionnaire he or she reports back to the Flemish Agency for Care and Health; all identifiable data related to the patient and the physician in question is then removed from the study database. A follow-up mailing of three reminders is performed 14, 28 and 42 days after the initial questionnaire was sent (following the Total Design Method [30]). 


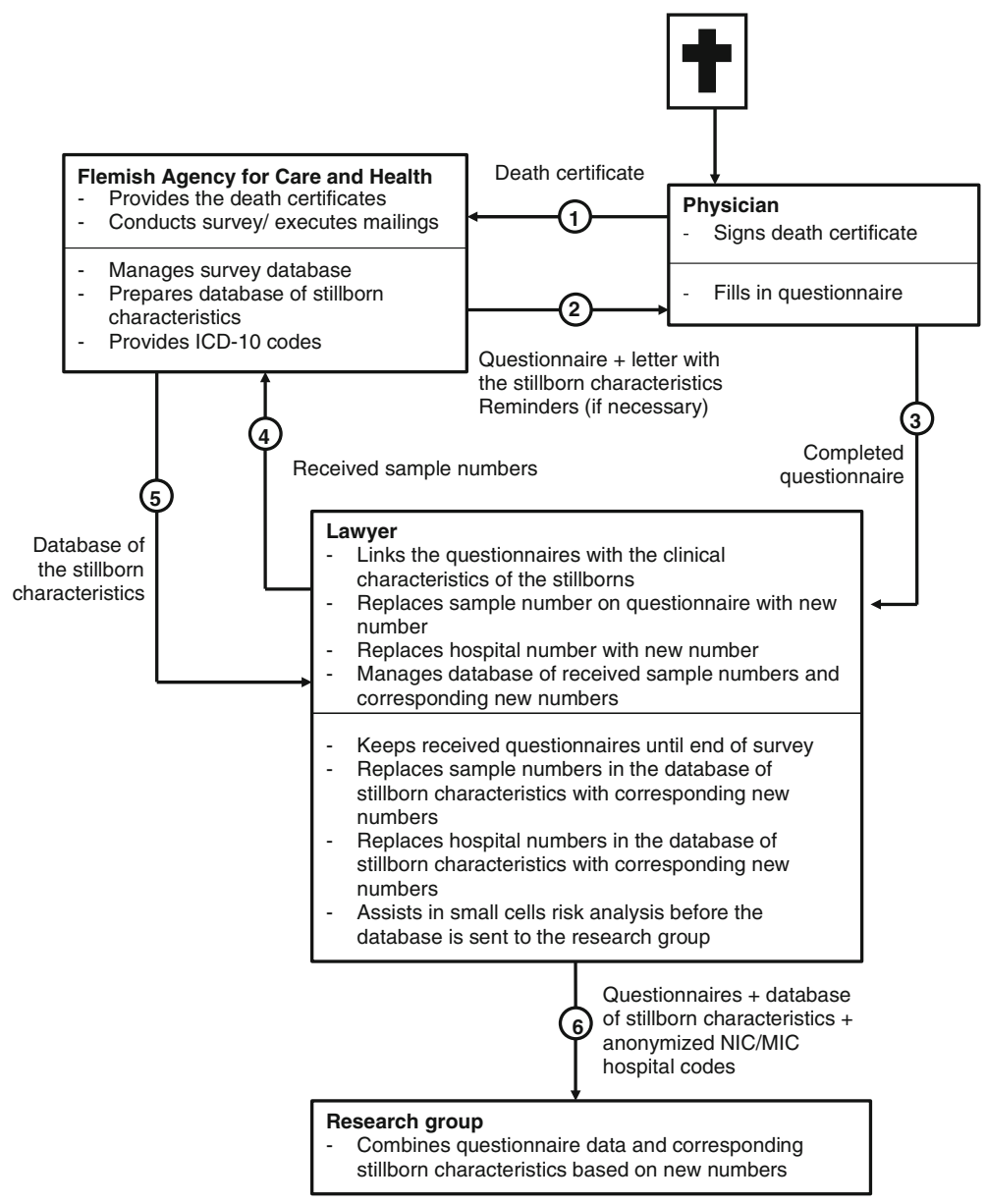

Fig. 1 Schematic overview of the mailing and anonymity procedure

2. The physician identifies the deceased or stillborn child based on the patient characteristics provided, fills out the questionnaires and returns these to a lawyer using a postage paid envelope. In case the certifying physician is not the treating or attending physician he or she is given specific instructions to pass the questionnaire to the treating physician if possible.

3. The lawyer, who is bound by confidentiality, safeguards the anonymity of the questionnaires. He or she codes the participating hospital wards so that comparisons can be made, and removes any possible identifying information of hospital, physician or patient, removes the unique numbers and reports these to the administration authorities. Additionally, place of death will not be sent to the researchers in order to ensure anonymity of the participating hospitals. The lawyer links the questionnaires with the information on the database from the death certificate administration authorities, and at the end of the data collection sends the linked database to the researcher group in which all identifiers will be removed and information can no longer be traced back to the corresponding death certificate.

4. The research group receives questionnaires and ensures that both in processing and analysing the database it will not be possible to determine the identity of the patient or the physician.

An alternative identification procedure for stillbirths between 22 and 26 weeks is included because the death certificate method proves to be challenging for stillbirths in that age group. Filling in death certificates of stillbirths between 22 and 26 weeks of gestation by a physician is not mandatory, which makes the death certificates a potentially incomplete sampling framework. We provided questionnaires to the ten biggest maternity wards in Flanders and the Flemish hospitals of Brussels so that physicians can fill out this questionnaire for every stillbirth from 22 weeks of gestation onwards and/or child with a birthweight from $500 \mathrm{~g}$ onwards. 
These maternity wards were chosen based on the presence of a NICU at the hospital, because of a high birth rate and/or because they are tertiary centres for prenatal diagnostics. For each stillbirth for which a questionnaire is completed, the physician is also asked to fill out a death certificate. This makes it possible for the lawyer to link the answers in the questionnaire to the clinical and demographic characteristics of the stillborn child (for a schematic overview of this procedure, see Fig. 2). The physician sends the questionnaire, together with a separate letter containing patient identification details to the lawyer and sends the certificate to the official death certificate agency. Because the latter sends patient identification details of death certificates for stillbirths to the lawyer, the lawyer can then determine whether a questionnaire has already been received for that death and notify the Flemish agency for Care and Health via email. In this case, no questionnaires are sent by the death certificate agency. The separate letter with patient identification details is destroyed as soon as the questionnaire is linked to the corresponding death certificate. If a physician did not fill out the questionnaire available in the maternity ward but did file a death certificate, they will still receive a questionnaire through the regular postal survey.

\section{Improving response rates}

To increase response, we follow the Total Design Method (TDM) [30]. Therefore, physicians will receive a maximum of three follow-up postal mailings. In order to further improve the response rate both in stillborns and in neonates and infants we will add an additional general follow-up. Every 3 to 4 months one of the researchers will visit all eight Flemish NICUs and all ten participating maternity wards to inquire about the course of the study. During visits, physicians will be able to ask

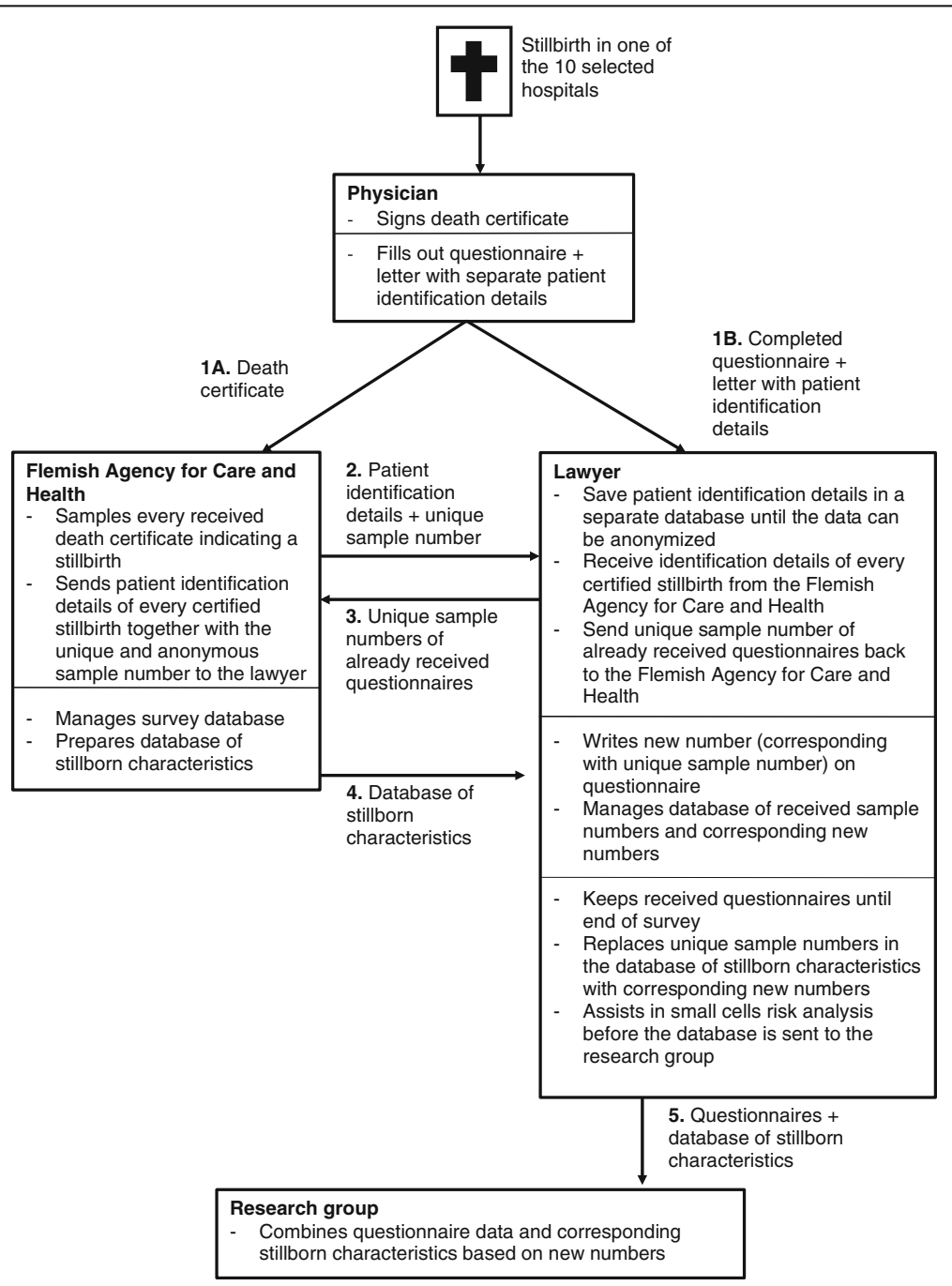

Fig. 2 Schematic overview of the parallel procedure in the ten biggest maternity wards 
questions, voice concerns or give general feedback. These visits are also meant to counter responder fatigue by stimulating motivation for the study duration. Furthermore, three consortium meeting will be organised to discuss the progress of the study with representatives of every NICU (one before the start, one half way through and one at the end of the study). Lastly, the study is also presented at relevant conferences and meetings.

\section{Ethical aspects and data protection}

The sensitivity of the research population and the delicate nature of our questionnaire makes it necessary to follow a rigorous ethical approval procedure. Ethics approval was obtained from the ethics committee of the University Hospital of Ghent and additionally from the Privacy Commission (CBPL), the Sectoral Committee of Social Security and Health, and the National Council of the Order of Physicians. For our parallel procedure in the ten biggest maternity wards, we obtained ethics approval from the ethics committees of all participating hospitals.

To ensure privacy and anonymity, as well as the precautions that have already be taken by using a lawyer, we strive to ensure full data protection. The data are always password protected and stored on a protected server. The database is not replicated or shared with third parties; all copies needed for analysis are destroyed afterwards.

\section{Data-analysis}

An SPSS 24.0 (SPSS Inc.) file is set up by the research group with a coding scheme for a certified data management company that will enter the data. The researchers will perform all data cleaning through SPSS syntax operations. Data will be analysed with descriptive statistics (valid percentages and 95\% confidence intervals), bivariate and multivariate association statistics.

\section{Discussion}

The objectives of this population study are to evaluate and monitor ELDs and their decision-making process in the foetal-infantile period including ELDs in the foetal and the neonatal period. This study design has several potential strengths as well as some limitations associated with the study population and the survey method.

\section{Strengths}

Our study is the first to examine foetal-infantile ELDs in their entirety. The results will broaden knowledge on which medical decisions are made in cases of congenital anomalies or severe disorders from the moment of viability, regardless of whether or not the child has been born. Even though ELDs have been researched both prenatally $[11,13,21]$ and in neonates $[14,16,31]$, the continuity of care and the overarching decision-making process has been missed in previous studies and therefore key elements (such as whether the ELD was made prenatally but performed after the child is born) could be overlooked.

Even though there are some studies comparing late TOP practices across European countries [11, 13, 21], not much is known about the full scope of end-of-life practices before birth (including non-treatment decisions) and their decision-making process. However, non-treatment decisions such as non-aggressive obstetric management with or without explicit intention to shorten the life of the foetus can also occur. One of the strengths of our study is therefore the inclusion of all types of possible ELDs in neonates and also before birth. Furthermore, even when the child died postnatally we inquire about decisions being made prenatally and thereby provide a full overview of ELDs without prior focus on one specific ELD.

Most research on ELDs in prenatal $[11,13]$ and neonatal $[6,17,20]$ settings is limited to single centre studies and based on reviews of medical records. Population-based studies based on officially registered death certificates, like ours, are however far more capable of obtaining robust data and reliable incidence rates since a nationwide scope ensures that the entire population is included. These could in turn lead to better understanding of current end-of-life care and detect points of improvement to benefit future parents and children with severe disorders. The only population-based study on Belgian neonatal ELDs dates back to 2000 [14] and since then, important societal changes such as questioning futile medical end-of-life care and refuting the idea of curative treatment as being necessarily beneficial could possibly have had an effect on end-of-life practice in unborn babies and neonates [32].

Aside from population specific strengths, some strengths can be attributed to the death certificate method in particular. These include international comparability, lack of patient burden and consequent attrition rates, reliability of the data, anonymity, and exclusion of possible selection bias by selecting certain physicians for the study. An overview of the strengths related to the death certificate method, which has successfully been implemented in adults [33], minors [26] and neonates [14], can be found in the research protocol of Chambaere et al. [29].

\section{Limitations}

One of the weaknesses of the study is that the death certificate method provides a challenge in the case of stillbirth between 22 and 26 weeks of gestation because completing a death certificate is not mandatory at this age. Despite our added data collection method, we 
cannot guarantee $100 \%$ coverage of stillbirths. Nevertheless, the reports from the Flemish centre of Perinatal Epidemiology, which registers every birth, will be available after the study and will make it possible to estimate the number of missing cases. Furthermore, despite the additional data collection method there is also no way to ensure that physicians will always complete a death certificate (as it is not obligatory), even when they fill out the questionnaire. It is therefore possible that we will receive questionnaires which we are not able to link to a death certificate which will therefore be unusable for this study.

Delays in the processing of death certificates can reach up to 4 months before the questionnaire is sent to the physician in the first method [29]. Therefore, a recall bias cannot be excluded. However, no other registration of deaths up to the age of 1 year exists and the only other registration of all births (live and stillbirths) occurs at the Flemish centre of Perinatal Epidemiology. This consists of fewer missing cases, however, and the delay in processing these documents can be up to 1 year which would drastically decrease the reliability of the responses. Furthermore, this method of registration is due to be merged with the existing death certificate registration, making our method the most reliable for future trend research.

We include all stillbirths from 22 weeks of gestation onwards because this is internationally acknowledged to be the limit of viability of the foetus $[24,27,28]$. However, some congenital anomalies can be detected before this viability threshold so we cannot exclude an ELD having been made before the 22 weeks cut-off used in this study. Furthermore, most Flemish neonatology wards only consider viability from 24,25 or even 26 weeks of gestation which could also have an impact on whether or not a death certificate is filled out.

\section{Implications for future research and practice}

Regular repetition of this study in the future is needed in order to monitor and evaluate changes in end-of-life practices in the foetal-infantile group. Because this study design allows application in other countries, we recommend international comparative studies to provide us with better insight into foetal-infantile end-of-life practices and incidence rates so that international foetal and neonatal care at the end of life can be optimised.

This can eventually aid the development of obstetrical, neonatal and paediatric guidelines to support an ethical end-of-life decision-making process.

\section{Abbreviations}

ELD: End-of-Life Decision; NICU: Neonatal intensive care unit; TDM: Total design method; TOP: Termination of pregnancy; WHO: World Health Organisation

\section{Acknowledgements}

We would like to thank the Flemish Agency for Care and Health, and the Brussels Health and Social Observatory. We are also deeply indebted to all physicians who participated in testing and validating the questionnaire, the participating hospitals (UZ Gent, UZ Brussel, UZ Leuven, UZ Antwerpen, Ziekenhuis Oost-Limburg Genk, GZA St Augustinus, AZ St Jan Brugge, ZNA Middelheim, St Lucas Gent, and AZ Groeninge Kortrijk), and to the lawyer and their personnel who act as a trusted third party between respondents and researchers. Lastly, we would like to thank Jane Ruthven for her language editing. Collaborators: Sabrina Laroche, Claire Theyskens, Christine Vandeputte, Luc Cornette, Hilde Van de Broek.

\section{Funding}

This study is funded by the Research Foundation Flanders (FWO) and the special research fund of Ghent University (BOF). K. Beernaert is Postdoctoral Fellow of the Research Foundation Flanders (FWO).

\section{Availability of data and materials}

Questionnaires and detailed research protocols (in Dutch) are available upon written request to the corresponding author (Laure.Dombrecht@UGent.be).

\section{Authors' contributions}

All authors contributed to the design and implementation of the study and to the writing of the manuscript. All authors take responsibility for the integrity of this manuscript. All information reported is honest, accurate and transparent. All authors read and approved the final manuscript.

\section{Ethics approval and consent to participate}

For the neonatal part of this study, approval was obtained from the Ethics Committee of Ghent University (Belgian Registration Number

B670201628795), the Privacy Commission (CBPL, registration number SA3/ VT005071970), the National Council of the Order of Physicians (registration number BD/wc/89997) and the Sectoral Committee of Social Security and health (registration number SCSZG/16/234). For the prenatal section of this study, approval was obtained from the Central Ethics Committee of Ghent University (Belgian Registration Number B670201730997), the Local Ethics Committees of the participating hospitals in Flanders, the Privacy

Commission (CBPL, registration number SA3NT005071970), the National Council of the Order of Physicians (registration number BD/wc/89997) and the Sectoral Committee of Social Security and health (registration number SCSZG/17/029). Sending back the questionnaire was seen as consent to participate.

Consent for publication

Not applicable.

Competing interests

The authors declare that they have no competing interests.

\section{Publisher's Note}

Springer Nature remains neutral with regard to jurisdictional claims in published maps and institutional affiliations.

\section{Author details}

${ }^{1}$ End-of-Life Care Research Group, Ghent University \& Vrije Universiteit Brussel (VUB), Ghent, Belgium. ${ }^{2}$ Department of Obstetrics, Women's Clinic, University Hospital Ghent, Ghent, Belgium. ${ }^{3}$ Department of Neonatology, Universitair Ziekenhuis Brussel, Vrije Universiteit Brussel, Brussel, Belgium. ${ }^{4}$ Department of Neonatology, Ghent University Hospital, Ghent, Belgium. ${ }^{5}$ Department of Development and Regeneration, KU Leuven, Leuven, Belgium. 'Division of Woman and Child, Clinical Department of Obstetrics and Gynecology, Fetal Medicine Unit, University Hospitals Leuven, Leuven, Belgium. ${ }^{7}$ Antwerp University Hospital, Antwerp, Belgium. ${ }^{8}$ Hospital Oost-Limburg Genk, Genk, Belgium. ${ }^{9}$ Hospital GZA St Augustinus, Antwerp, Belgium. ${ }^{10}$ AZ St Jan Brugge, Brugge, Belgium. "'ZNA Middelheim, Antwerp, Belgium. 
Received: 27 February 2018 Accepted: 9 July 2018

Published online: 03 August 2018

\section{References}

1. Rüegger C, Hegglin M, Adams M, Bucher HU. Population based trends in mortality, morbidity and treatment for very preterm- and very low birth weight infants over 12 years. BMC Pediatr. 2012;12(1):17. https://doi.org/10. 1186/1471-2431-12-17.

2. Devlieger R, Martens E, Martens G, Van Mol C, Cammu H. Perinatale activiteiten in Vlaanderen 2014. Stud voor Perinat Epidemiol. 2014;28:56.

3. Barfield WD, Committee on Fetus and Newborn. Standard terminology for fetal, infant, and perinatal deaths. Pediatrics. 2016;137(5):e20160551. https:// doi.org/10.1542/peds.2016-0551.

4. Cuttini M, Nadai M, Kaminski M, et al. End-of-life decisions in neonatal intensive care: physicians' self-reported practices in seven European countries. EURONIC Study Group. Lancet. 2000;355:2112-8.

5. Verhagen E, Sauer PJJ. The Groningen protocol--euthanasia in severely ill newborns. N Engl J Med. 2005;352(10):959-62. https://doi.org/10.1056/ NEJMp058026.

6. Berger TM, Hofer A. Causes and circumstances of neonatal deaths in 108 consecutive cases over a 10-year period at the Children's Hospital of Lucerne, Switzerland. Neonatology. 2009;95(2):157-63. https://doi.org/10. 1159/000153100.

7. Provoost V, Deliens L, Cools F, et al. A classification of end-of-life decisions in neonates and infants. Acta Paediatr. 2007;93(3):301-5. https://doi.org/10 1111/j.1651-2227.2004.tb02950.x.

8. Bijma HH, Schoonderwaldt EM, Van Der Heide A, Wildschut HIJ, Van Der Maas PJ, Wladimiroff JW. Ultrasound diagnosis of fetal anomalies: an analysis of perinatal management of 318 consecutive pregnancies in a multidisciplinary setting. Prenat Diagn. 2004;24(11):890-5. https://doi.org/10. 1002/pd.883.

9. Bijma HH, van der Heide A, Wildschut HI. Decision-making after ultrasound diagnosis of fetal abnormality. Reprod Health Matters. 2008;16(31 SUPPL): 82-9. https://doi.org/10.1016/S0968-8080(08)31372-X

10. Chervenak FA, McCullough LB. Nonagressive obstetric management: an option for some fetal anomalies during the third trimester. JAMA. 1989; 261(23):3439-40.

11. Papiernik E, Zeitlin J, Delmas D, et al. Termination of pregnancy among very preterm births and its impact on very preterm mortality: results from ten European population-based cohorts in the MOSAIC study. BJOG An Int J Obstet Gynaecol. 2008;115(3):361-8. https://doi.org/10.1111/j.1471-0528. 2007.01611.x.

12. Bosma JM, van der Wal G, Hosman-Benjaminse SL. Late termination of pregnancy in North Holland. Br J Obstet Gynaecol. 1997;104(4):478-87.

13. Garne E, Khoshnood B, Loane M, Boyd PA, Dolk H. Termination of pregnancy for fetal anomaly after 23 weeks of gestation: a European register-based study. BJOG An Int J Obstet Gynaecol. 2010;117(6):660-6. https://doi.org/10.1111/j.1471-0528.2010.02531.x.

14. Provoost V, Cools F, Mortier F, et al. Medical end-of-life decisions in neonates and infants in Flanders. Lancet. 2005;365(9467):1315-20. https:// doi.org/10.1016/S0140-6736(05)61028-8.

15. Vrakking AM, Van Der Heide A, Onwuteaka-Philipsen BD, Keij-Deerenberg IM, Van Der Maas PJ, Van Der Wal G. Medical end-of-life decisions made for neonates and infants in the Netherlands, 1995-2001. Lancet. 2005;365(9467): 1329-31. https://doi.org/10.1016/S0140-6736(05)61030-6.

16. ten Cate $K$, van de Vathorst S, Onwuteaka-Philipsen BD, van der Heide A. End-of-life decisions for children under 1 year of age in the Netherlands: decreased frequency of administration of drugs to deliberately hasten death. J Med Ethics. 2015;41(10):795-8. https://doi. org/10.1136/medethics-2014-102562.

17. Roy R. Decision making and modes of death in a tertiary neonatal unit. Arch Dis Child Fetal Neonatal Ed. 2004;89(6):F527-30. https://doi.org/10. 1136/adc.2003.032912

18. Barton $\mathrm{L}$. The contribution of withholding or withdrawing care to Newborn mortality. Pediatrics. 2005;116(6):1487-91. https://doi.org/10. 1542/peds.2005-0392.

19. Verhagen $A A E$, Janvier $A$, Leuthner $S R$, et al. Categorizing neonatal deaths: a cross-cultural study in the United States, Canada, and the Netherlands. J Pediatr. 2010;156(1):33-7. https://doi.org/10.1016/j.jpeds. 2009.07.019.
20. Cuttini M. The European Union collaborative project on ethical decision making in neonatal intensive care (EURONIC): findings from 11 countries. J Clin Ethics. 2001;12(3):290-6.

21. Boyd PA, DeVigan C, Khoshnood B, et al. Survey of prenatal screening policies in Europe for structural malformations and chromosome anomalies, and their impact on detection and termination rates for neural tube defects and Down's syndrome. BJOG An Int J Obstet Gynaecol. 2008;115(6):689-96. https://doi.org/10.1111/j.1471-0528.2008.01700.x.

22. Chambaere K, Bilsen J, Cohen J, Onwuteaka-Philipsen BD, Mortier F, Deliens $\mathrm{L}$. Trends in medical end-of-life decision making in Flanders, Belgium 19982001-2007. Med Decis Mak. 2011;31(3):500-10. https://doi.org/10.1177/ $0272989 \times 10392379$

23. Pinter $A B$. End-of-life decision before and after birth: changing ethical considerations. J Pediatr Surg. 2008;43(3):430-6. https://doi.org/10.1016/j. jpedsurg.2007.09.054.

24. Rennie JM, Leigh B. The legal framework for end-of-life decisions in the UK. Semin Fetal Neonatal Med. 2008;13(5):296-300. https://doi.org/10.1016/j.siny. 2008.03.007.

25. Provoost V, Deliens L, Cools F, et al. A classification of end-of-life decisions in neonates and infants. Acta Paediatr. 2004;93(3):301-5. https://doi.org/10. 1080/08035250410022954

26. Pousset G, Bilsen J, Cohen J, Chambaere K, Deliens L, Mortier F. Medical end-of-life decisions in children in Flanders, Belgium. A population-based postmortem survey. Arch Pediatr Adolesc Med. 2010;164(6):547-53. https:// doi.org/10.1001/archpediatrics.2010.59.

27. Boland RA, Davis PG, Dawson JA, Doyle LW. Outcomes of infants born at 22-27 weeks' gestation in Victoria according to outborn/inborn birth status. Arch Dis Child-Fetal Neonatal Ed. 2017;102(2):F153-61. https://doi.org/10. 1136/archdischild-2015-310313.

28. Fischer N, Steurer MA, Adams M, Berger TM. Survival rates of extremely preterm infants (gestational age \&lt;26 weeks) in Switzerland: impact of the Swiss guidelines for the care of infants born at the limit of viability. Arch Dis Child Fetal Neonatal Ed. 2009;94(6):F407-13. https://doi.org/10.1136/adc. 2008.154567.

29. Chambaere K, Bilsen J, Cohen J, et al. A post-mortem survey on end-of-life decisions using a representative sample of death certificates in Flanders, Belgium: research protocol. BMC Public Health. 2008;8(1):299. https://doi. org/10.1186/1471-2458-8-299.

30. Hoddinott SN, Bass MJ. The dillman total design survey method. Can Fam Physician. 1986;32:2366-8.

31. Provoost V, Cools F, Bilsen J, et al. The use of drugs with a life-shortening effect in end-of-life care in neonates and infants. Intensive Care Med. 2006; 32(1):133-9. https://doi.org/10.1007/s00134-005-2863-2.

32. Carpentier N, Van Brussel-De Vriendt L. On the contingency of death. A discourse-theoretical perspective on the construction of death. Crit Discourse Stud. 2012;2(9):99-116.

33. Bilsen J, Cohen J, Chambaere K, et al. Medical end-of-life practices under the euthanasia law in Belgium. N Engl J Med. 2009;361(11):1119-21. https:// doi.org/10.1056/NEJMc0904292.
Ready to submit your research? Choose BMC and benefit from:
- fast, convenient online submission
- thorough peer review by experienced researchers in your field
- rapid publication on acceptance
- support for research data, including large and complex data types
- gold Open Access which fosters wider collaboration and increased citations
- maximum visibility for your research: over $100 \mathrm{M}$ website views per year
At BMC, research is always in progress. 